\title{
The Emerging Requirement for Digital Addiction Labels
}

\author{
Raian Ali, Nan Jiang, Keith Phalp, Sarah Muir, John McAlaney \\ Faculty of Science and Technology, \\ Bournemouth University, United Kingdom \\ \{rali, njiang, kphalp, swilliams, jmcalaney\}@ bournemouth.ac.uk
}

\begin{abstract}
Context \& motivation] Digital Addiction, e.g. to social networks sites and games, is becoming a public interest issue which has a variety of socio-economic effects. Recent studies have shown correlation between Digital Addiction and certain negative consequences such as depression, reduced creativity and productivity, lack of sleep and disconnection from reality. Other research showed that Digital Addiction has withdrawal symptoms similar to those found in drug, tobacco, and alcohol addiction. [Question/problem] While industries like tobacco and alcohol are required by certain laws to have a label to raise awareness of the potential consequences of the use, we still do not have the same for addictive software. [Principal ideas/results] In this study, we advocate the need for Digital Addiction labels as an emerging ethical and professional requirement. We investigate the design of such labels from a user's perspective through an empirical study, following a mixed-methods approach, and report on the results. [Contribution] Our ultimate goal is to introduce the need for labelling to both researchers and developers and provide a checklist of questions to consider when handling this non-functional requirement.
\end{abstract}

Keywords: Digital Addiction, Ethical and Professional Requirements, NFR

\section{Introduction}

Digital Addiction (hereafter DA) is becoming a serious issue which has a variety of consequences such as reduced involvement with their real life communities [1] and lower Grade Point Averages due to its negative impact including procrastination, distraction, and poor time-management [2]. People who feel insecure in real life often try to compensate in the digital world [3]. When that later option fails, it reduces even more their self-confidence and self-esteem [4]. Studies showed that addiction to Facebook has a negative impact on romantic relationships (leading to divorce in some cases) due to disclosure of private information, cyber-stalking and electronic surveillance by one's partner [5].

Young [6] classifies online addiction into five types: Computer (games) addiction, Information overload, Net compulsions, Cyber-sexual addiction, and Cyberrelationship addiction. Social network addiction, which is relatively new, would map to the last category but it may still include elements of the others, e.g. games. Such DA has characteristics similar to those found in "traditional" addiction such as mood modification, salience, tolerance, withdrawal symptoms, conflict, and relapse [7]. This paper will take social networks and games as exemplar addictive software. 
However, in spite of this increasing recognition of the phenomenon of DA, there is still not enough data to decide whether the medium, in this case software and online space, has the main responsibility for addiction or whether personal characteristics is the genuine source and the medium is just a facilitator or a tool $[4,5,7]$. That is, the fundamental reason, at least in the beginning, may not be necessarily the subject of addiction, i.e. the software in our case. In all cases, awareness that a medium could facilitate an addictive behaviour should be a moral requirement if not a legal one.

DA is still an under-researched concept especially in the software engineering community. In our previous work [8], we defined DA from a requirements engineering perspective as "the excessive use of certain software-mediated operations to reach certain requirements. This includes the case when the use itself is compulsive or impulsive and also the case when the user cannot switch to other available alternatives to reach the same requirements without a good reason". The impulsive and compulsive use could lead to unconscious and hasty actions, which exacerbate the consequences and necessitate even more a sort of warning and awareness messages.

In this paper we explore the responsibility of the software industry in raising awareness of the potentially addictive nature of their products. Unlike industries like tobacco ${ }^{1}$ and alcohol, which are required by law to raise such awareness through labels, software is still not seen subject to such social and ethical requirement. We confirm and enhance our initial argument and discuss thoughts on utilising the perception of users to best design DA labels. We follow a mixed-methods approach starting with a qualitative interview-based phase and following with a quantitative surveybased phase. We draw conclusions that we believe will inform further research on the topic and draw attention to this emerging professional non-functional requirement.

The paper is structured as follows. In Section 2 we describe the study design phases. In section 3, we report on the results of the first phase together with the degree of agreement of the larger samples involved in the quantitative phase. In Section 4, we present a set of research challenges with regard to the engineering of the labelling requirement. We conclude the paper and present future work in Section 6.

\section{The Study Design}

We study DA labels from a user perspective. Users are the ultimate target of such warning or awareness messages and thus their perspective is premium. Labelling is different from controlling. Labelling is to raise awareness and aid certain perceptions and behaviour change. Thus, it is a sort of recommendation similar to the Nudge approach to behaviour change in the health and social field where the recommender attempts to encourage people to make a better choice but do not actually attempt to control them. An example is to put health warnings on cigarettes but not make smoking illegal [14]. In [9] we conducted a study to understand how users would like recommendation messages to be delivered. The study concluded that besides the basic feature of having a relevant message in terms of content and presentation, users require three other features: control, awareness, and adaptivity. These features guided the design of our interview questions and the following survey quantitative phase.

1 http://www.tobaccolabels.ca 
- Message. Users would like to see relevant content, i.e. content which addresses their particular needs rather than generic messages. The presentation and the medium used to deliver the content are an integral part of the message.

- Control. Users would like to be able to specify when and how a label should be shown and when to leave that decision to software. This includes the content, the presentation, the time and the inference strategy software follows to come up with a suitable label.

- Awareness. Users would like to be informed of the reasons why a label is being shown to them and what was collected about them to form it. It is a mixture of privacy and curiosity concerns. In other words, this relates to the meta-data users would like to know about the label and its development process.

- Adaptivity. Although users would like a certain degree of control, they would still like software to reason on their behalf and choose the best way to deliver the labels according to their dynamic context.

11 participants, five male and six female, aged between 19 and 35 years old, were recruited for the interview where four were professionals and seven were students studying Computing (four) and Psychology (three). Seven of them were selected after a pre-selection survey as they were all flagged up after the survey that they felt they would like to be assisted by labelling and warning messages to control their usage. For counter balancing, four participants, who did not feel the labelling was an efficient idea, were also invited to be interviewed. These four participants gave us an idea of what could be obstacles to achieving users' acceptance of DA labels. Each interview lasted about 30 minutes and the conversation was audio recorded and transcribed after acquiring the consent from the participants.

To confirm and enhance the results obtained through the content analysis of the interview, we designed a survey of nine questions, each covering some of the findings related to the Content, Presentation, Control, Awareness and Adaptivity of the DA labelling. The survey was disseminated through mailing lists to students at Bournemouth University, BCS-HCI mailing list, the social media and mailing lists of the authors. The survey started with a test question informed by the CAGE questionnaire [15] to detect whether a participant has any sort of addictive usage. 16 participants did not pass the test questions and their survey was terminated. 72 participants completed the survey ( 35 male, 36 female, and one preferred not to say). The age bands distribution was 18-25 (47\%), 26-34 (33\%), 35-44 (6\%), 45-54 (4\%), 55-64 (8\%), 65+ (0\%), and $2 \%$ preferred not to answer. The survey was tested on three participants before being disseminated.

\section{$3 \quad$ Findings}

In addition to the four facets of the DA message described in Section 2, we also aimed to get users' general view of the concept. This is due to the novelty of the concept itself, which makes the investigation of its feasibility and potentials important per se before delving into the details of how it should be developed. 


\subsection{General view}

The term Digital Addiction did not raise any concerns to any of the interviewees and survey participants. The interview participants felt that DA is a sort of addiction although it may not have the same physiological consequences as substance addiction. Interviewees emphasized the social and mental well-being as the area in which DA has major effects. However, we are still unsure whether people will still like to be called "addicts" in the labels directed to them. One participant warned that the term may put some people off and may make them react negatively unless an appealing argument is made for their particular usage. $32 \%$ of the survey participants thought DA labelling is certainly needed, 50\% thought is likely a good idea, $15 \%$ thought it is unlikely to be useful and 3\% thought it is not going to work. This shows the high potential of investigating the topic.

Interestingly, according to the participants' comments, addiction is not only that related to the excessive usage, compulsively and/or impulsively, it is also about the actions a person would do in a hasty and non-thoughtful style with little resistance to the temptation for that. E.g. in a party a user may take pictures and post without thinking of the consequences. The ease of the process and the speed and the scale of information spread become over-attractive and encourage hasty actions to certain people. In addition to this observation, DA in its intuitive sense of over-spending time on digital media is not necessarily to achieve some sort of entertainment (called infotainment). DA may be caused by the fact that users feel the need to be online all the time to ensure the sanity of their social presence, e.g. no one is annoyed by their posts or felt ignored if they did not respond.

The concept of DA labelling is seen a powerful tool for a number of reasons. The first is that many people are simply unaware of how much they use social networks and games. A warning message would inform them in the first place even without any other content other than the time they spent. The label is also needed when there is a significant risk that the usage interrupts and distracts other activities, e.g. students having a "cyber-break" during a lecture. The label has particular value when users are unaware of potential withdrawal symptoms. E.g. some people feel lost without their connection to their social network or gaming community which could often happen not only because of technical errors but also due to social reasons, such as being banned by a group, or losing online fans, etc. For certain vulnerable groups, e.g. children, the label is not only important for the user but also for their carer. Finally, although it is generally agreed that software, like social networks and games typically aim to attract more users, labelling remains a moral and ethical responsibility which will inspire users trust in the software and increase their loyalty.

In spite of that, certain cases would hinder the feasibility and meaningfulness of such a label. Some of our participants emphasized that it is not purely a decision of the individuals to control their usage when everyone else is using it and they need to react. This means DA label targeting and advising the individuals needs to be aware of that. DA is in part a collective responsibility. One interviewee said that "if everyone posts what is needed only, people would not feel the need to check often and spend much time. It is like offering a person a drink". It is also stated that the label is 
secondary to the software design itself. It would be seen awkward to have a very tempting design and then show a label warning of the consequences of use. This view argues that it will be more sensible to embed the control of use in the design itself and aid users technically in moderating their excessive use instead of the labels. In an extension to that, DA label should not mean exempting developers from the responsibility of a deliberate inclusion of addictive elements in their software. Finally, a label is seen as ineffective when the original reason for DA is more than a careless usage. Depression and tension could lead to people spending hours and hours on games and social networks. Warning messages would look like a noise in that case. Similarly, introverts in real life could find compensation online and their excessive usage is the norm where their engagement in real-world in-person is seen the exception. Labels may mean little to them.

An interesting observation about DA label is the possibility to turn it to a social label in two ways. It could compare to other users who agreed to share their statistics of usage and, also, it could be generated by colleagues instead of software. This is similar to the case when friends try to warn someone to stop drinking in a party. There is still a space for dual use here which needs research, e.g. competing on drinking more.

\subsection{Message: Content and Presentation}

Regardless of the information content and the way it is presented, it is generally agreed that positive and gentle approach should be followed until it is an extremely excessive usage. This positive labelling will not put users off so they may stop the usage of the software all together. An encouraging approach would not leave a negative effect on the self-esteem, e.g. feeling over-guilty especially those who are unaware of the whole concept. Finally, judging a person usage to be a sort of addiction is an approximation so it may be wise to avoid confirmation and being so strict. In the following, we list the elements which could form the content of DA messages. Throughout the paper, the percentages represent the number of survey participants who ticked the option

\section{Usage Related:}

- Time already spent on the software $(86 \%)$

- The number of times I checked/visited the software $(56 \%)$

- Usage "bill", like mobile bills and bank statements (47\%)

- The features which I heavily used (e.g., Like, tagging, messaging etc.) (17\%)

\section{Consequence related:}

- Consequences on real social life (e.g., relations breakdown) (51\%)

- Effects on physiological and mental health (e.g., eye strains, tension etc.) (50\%)

- Damage on your public profile (potentially seen by employer, etc.) (39\%)

- The ease and speed of information spread once shared (32\%)

- Potential risks on you, e.g. when you use social networks in excessive, hasty and unthoughtful way (29\%)

- Consequences on your on-line relationship with others (e.g., hasty and not thoughtful interactions could be misinterpreted etc.) (29\%) 
- Consequences on online contacts (e.g., hasty and excessive tagging and sharing could affect the privacy of people involved in the posts) $(19 \%)$

\section{Advice related:}

- Suggestion/advice on potentially interesting real life activities based on your usage, e.g. going to a social event which matches your detected online interests (44\%)

- Factual and proved statements about the benefits of regulating usage styles (38\%)

- Suggestions/advice on how to regulate the usage style, e.g. using filters to reduce the amount of feeds/notifications (33\%)

By analysing the comments in both phases, the features offered by software and cause DA falls into four categories which are similar to the game elements categories proposed by Bartle's specification explained in [10].

- Achievement: when the software feature drives the user to achieve more, e.g. users who keep checking and posting to increase their rank and social capital.

- Exploration: when the feature keeps the degree of curiosity high and drives the person to keep connected to know what is next.

- Socializing: when the wealth of connectivity features and ease of access make one overly a socializer. This is sometimes due to a sort of escalating commitments where people are online, not because of the pleasure, but to see what others would require from them and whether they upset someone or got negative comments.

- Killing: which is more obvious in the case of gaming where a mental satisfaction and stress relief are achieved when one causes harm, virtually, to someone else.

On top of the four features, there are meta-features that increase the addictive nature of software. This includes the ease of use, the real-time nature, the scale of communication and vast diversity of information in an easily navigable cyberspace. These features mean additional attraction which would encourage addictive usage style.

In terms of presentation, DA messages could be delivered via various modes. Each one depends on different factors such as the device used, the activity being done, the stage of addiction and the personality type. We identified eight ways such messages could be delivered. The percentages reflect the amount of survey users who wanted it. An investigation of when to use each delivery method is still to be researched:

- Time-based progress status (e.g., clock/timers for your usage amount) (61\%)

- Dynamic colouring of interfaces to reflect your degree of usage (e.g., Green bar for reasonable use, Red bar for excessive use, etc.) (53\%)

- Pop-up notifications (44\%)

- Personalised metaphors (e.g., an avatar of you when being overly engaged) (31\%)

- Hardware based interactions (e.g., vibration and flickering on mobile phones or 3D glasses of gamers) $(26 \%)$

- Sounds (e.g., beeping when you overly play a game or check Facebook) (21\%)

- Offline notifications, e.g. sent as a message or email (19\%)

- Analogy to traditional addiction (e.g., a metaphor of consumption of number of "digital" alcohol glasses) (18\%) 
The participants emphasized a number of characteristics DA labels should enjoy in order to have a positive reaction from the users. This included:

- Supportive content (61\%). DA labels may not be necessarily in the form of a warning form. For example, when losing a game, a user would like a message moderating that feeling which will reduce the desire to start another round. Encouraging the healthy use is also part of this case.

- Non-repetitive content (54\%). Users will tend to ignore DA labelling if when it issues messages with similar content and presentation style.

- Not overly-negative content (51\%). Users do not like to be overly-warned as this could lead to disrupt their healthy usage. This is similar to the case where gambling is overly associated with people losing their properties and savings, time and social position while it is still possible that people use it moderately as an entertainment tool.

- Socially-generated content (36\%). It appeared that messages could be made by friends and this would increase their exciting nature for some users. It is similar to the case when one receives a friendly comment to stop drinking. Similarly the software would need to offer friends to do the same.

- Precautionary content (36\%). This is a proactive approach to DA labels. E.g. the message may not be about the current excessive use but the potential to get it or about the high-dependency on software which may lead to serious consequences when lost, e.g. when the connection or a password is lost, a page is closed, a membership is terminated, etc.

\subsection{Control}

The control aspect relates to what decisions users would like to have over DA labelling and how to express that to software. As a general principle, the interviewees emphasized that labelling, and following what is suggested in it, should remain a choice of the user (or a carer in the case of minors). Unlike alcohol and tobacco, the label could also trigger mechanisms to react to an addictive usage style, e.g. blocking for few hours or reducing some features such as limiting the amount of posts one can make per day. Although this is possible, interview participants' agreed that a user would always find a way, probably other software, when overly warned and controlled. Thus, we excluded this option and we would consider it part of a more like parental control than warning.

In terms of What, users would like to be able to control. $36 \%$ of the survey participants would like the software to be highly autonomous in forming and delivering labels once they enabled the labelling service. Still, the participants have preferences about being able to control the various settings of labelling:

- The frequency of sending labels $(60 \%)$,

- How the label should be presented (graphics, sound, email, etc.) (50\%),

- The time(s) the label should be delivered (44\%),

- The actions that trigger a label (e.g., the things when used/done would require a generation and delivery of a label) $(40 \%)$,

- The type of information the label could contain (39\%), 
- The accepted sources of the label (e.g., accept labels designed by certain developers, institutions or people) (38\%),

- The strategy through which the labelling is decided (proactive or reactive to my usage style, comparative/relative to others or absolute) (31\%).

In terms of How, it seems there is a degree of complexity in how users makes decisions. Besides the technical complexity of deciding what to control and what to leave to software, users have a paradoxical requirement to be looked after by a trusted hand, whether software or friends, but without being controlled and overly warned at the same time. An interviewee gave an interesting analogy saying that "it is similar to the feeling when one takes the bottle of wine from you because you have to drive or to go to work the day after". This point could be divided into two facets:

- Resistance to change. This is a challenge to handle during the initial period of issuing the labels. An analogy to that is the resistance to tobacco labels and smoking designated areas. Interviewees expected that, by time, people will accept DA labels as part of the new online norms. From engineering perspective, the management of that change is a socio-technical problem which is exacerbated by the current view of social media of being an indicator of trendiness by many people. This is analogous to the stereotype of smokers and drinkers in old marketing adverts and classical movies till laws prohibited that considering it a sort of manipulation.

- Calibrated agreement. Users tend to accept software to take decision on issuing DA labels and even enacting some sort of precautionary procedures, e.g. coolingoff period, when trust is established. Interviewees indicated that they would like to start with a clear separation of decisions and calibrate the relation over time so that a mutual understanding between their personal preferences and software reasoning is eventually established. This is similar to a supervision relationship, in business and academia, which calibrates overtime and eventually yields a mutual agreement through a sort of natural selection of a range of attitudes and treatments. The importance of this comes from the need to avoid making DA labelling specification a burden on users' experience which is the focus of the rest of this section.

In terms of the technical specification of DA labels, interviewees indicated the need for a range of facilities which would make labelling both accepted and efficient. Interviewees indicated that they are already involved in a plenty of other configurations, such as security and privacy, and adding yet another one for DA labels will be another threat to their comfortable usage. This is not only about setting up the labelling configuration but also the worry of how this would work given the newness of the concept. We identified various aspects on DA label specification:

- Time-based specification. The basic form of permission given to software, in relation to issuing the DA label, is time-based. This includes both the time to issue the label, e.g. when users exceed a certain time, and the time to present it, e.g. once users $\log$ in/out. The threshold is not necessary on the amount of time and the amount of checks but also on the nature of the actions. It is also context dependent, e.g. "extra use" meaning in holidays is different from it in work days. 
- Features-based specification. Certain features of software seem to be highly addictive and lead to a hasty and excessive usage style, which, in turn, could also lead to consequences not only for the user but also their community. Users appreciate the control given to the software in issuing the warning label in relation to those features. E.g. sharing and tagging are examples of features where people may feel unable to control at certain point. An interviewee mentioned that "it is so easy and tempting to press the button, but once it goes you may not be able to retract".

- Complementary actions. The label should be seen as a part of an integrated process of a usage regulation, i.e. it is not a standalone treatment. This is especially true in the case of social software where there could be social consequences of not being online and such as losing attention and being missed when needed. The label should be complemented with supplementary procedure, which avoids the case that the new usage style harms the basic requirements, e.g. turning the profile message to explain absence to close friends. These actions could be part of the configuration and control of DA labels which make the process more holistic.

- Social Control. An interesting observation is that people would accept warning messages to come from their friends and contacts. This is not necessarily done on a one-to-one or direct basis. A person could generate messages to be shown to all friends or group members who exceed a certain limit. Although this idea seems futuristic, it stimulated interesting discussions. Some participants indicated that groups could nominate a guard or agree on norms of usage and DA labels should follow that mutual agreement and could be shown as a positive social pressure. This is a sort of blended control where software, users and the group take part of it.

- Specification reuse. Specifying DA labels and how this should be inferred and presented would add additional overhead. The idea of reusing labelling patterns suggested by trusted social entities, such as health institutes or close friends, seems to be interesting. This is similar to eating style proposed by reputed diet specialists.

\subsection{Awareness}

In [9], we showed that people would like to know why a certain recommendation is being delivered to them at a certain time. This is mainly for privacy and curiosity concerns and others sorts of meta-data to describe the label. People would like to know, or be offered to know, how the DA labels are processed for them and why a message is presented in a certain format and language. This becomes more of an issue when trust has not been established yet and when labelling is conducted as social activity, e.g. recommendations coming from colleagues as described in 3.3.

A general view is that the usage of online software, especially social networks, entails that certain usage data are collected about the user. Interviewees agreed that seeing a label warning about a usage style does not raise unusual privacy concerns in comparison to those already recognized. For example, it is commonly known that data about the amount a user spends on a certain page are sometimes used to infer the market trends and preferences of certain populations. However, in the case of the labelling, and given the fact it is typically to aid users, these typical concerns could have consequences on the efficiency and acceptance of the label itself and users experience with the software in general. The following points elaborate that further. 
- Trust. People would start to question how DA labels were inferred when the software does not inspire their trust in the first place. Building a trust relation seems to be superior to warning the user of their addictive usage, no matter how right the warning is. Participants indicated that, besides the baseline trust with the software as a whole, the labelling-related trust is typically built through (i) factual correctness, e.g. by giving accurate and unbiased information, (ii) treatment, e.g. the language used and (iii) transparency of inference, i.e. the possibility to see what and how data were used. Some users would increase their level of trust in the software when they become aware that it is careful about them and it is putting its popularity at risk (knowing that they may simply leave it) to keep their usage within healthy levels. That is, the message would need to convey this caring attitude.

- Loss of relationship. As a continuation of the previous point, labelling could lead to a loss of closeness between users and software. Some participants stated that at times they may feel someone is picking on them. It is important to choose the right wording and graphics, e.g. loss vs. gain framing [11]. Being positive seems to moderate the negative feeling of being overly unhealthy at least for those who are unaware of the whole concept of DA and its potential consequences.

- Moderate labelling. The users should be made aware of the approximate and potentially imprecise nature of the label. Unlike tobacco and alcohol, where the consequences are pretty tangible and measurable, the consequences of DA are not necessarily visible and may not be the same for every person. The consequences are to a large extent related to the social and mental well-being rather than physiological. Awareness of the users about the approximate nature of the label is also an ethical principle, at least currently, due to the lack of experiments on DA.

- Informed consent, iteratively. People should be made aware that DA labelling is going to be installed and they should have the right to choose or exclude it. An explanation of the data which are going to be collected will inspire trust. For each label, a link to the stats which explain how the label was generated will be likely appreciated. Although this may not be interesting to many, but the fact it exists will increase trust and transparency and it will be consistent with the fact that labelling is ultimately an ethical and professional practice.

- Indirect disclosure. Users should know how their usage data will inform the design of others labels, e.g. when calculating the usage of an average user. This may not be always clear. For example, when a label contains a comparison of a person usage to the friends in a certain group, this in part implies how that group is collectively using it. This, in some cases, could mean much knowledge about individuals. In the case of social control of labelling the situation becomes paradoxical where a user would need to balance between giving the group some control over the labelling and being at the same time reserved on sharing usage information.

- Developers' awareness. Another interesting point was raised about the need to educate developers themselves to appreciate the dual usage of their software product. Their awareness will be the first step before they can raise users' awareness. $70 \%$ of the survey users believed that this is not a main concern of developers. Some survey participants who disagreed or remain neutral gave a contradicted view saying that making the software addictive is a deliberate goal in certain cases. 
Table 1 shows the survey results on the same aspect (SA: Strongly Agree, A: Agree, N: Neither Agree nor Disagree, D: Disagree, SD: Strongly Disagree).

Table 1. Users view on the awareness dimension of DA label

\begin{tabular}{|l|l|l|l|l|l|}
\hline \multicolumn{1}{|c|}{ Statement } & SA & A & N & D & SD \\
\hline Software needs to inspire my trust before I accept labelling. & $31 \%$ & $54 \%$ & $13 \%$ & $3 \%$ & $0 \%$ \\
\hline $\begin{array}{l}\text { Labelling may lead to less natural use of software and make me } \\
\text { lose closeness with it (no matter how useful labelling is). }\end{array}$ & $4 \%$ & $26 \%$ & $44 \%$ & $22 \%$ & $3 \%$ \\
\hline $\begin{array}{l}\text { Software can only have approximation and estimation about my } \\
\text { usage, so it should always make labels less confirmatory. }\end{array}$ & $4 \%$ & $50 \%$ & $35 \%$ & $8 \%$ & $3 \%$ \\
\hline $\begin{array}{l}\text { I should be able to know how the label was generated and why; } \\
\text { this will increase my acceptance of it. }\end{array}$ & $25 \%$ & $58 \%$ & $8 \%$ & $6 \%$ & $3 \%$ \\
\hline $\begin{array}{l}\text { I need to be able know how my usage data and reactions to } \\
\text { labels are used even if this is to enhance the labelling service. }\end{array}$ & $14 \%$ & $54 \%$ & $22 \%$ & $8 \%$ & $1 \%$ \\
\hline $\begin{array}{l}\text { I feel software developers/industries are often unaware of, or } \\
\text { uninterested in, the addictive nature of their software and its } \\
\text { consequences }\end{array}$ & $24 \%$ & $46 \%$ & $11 \%$ & $11 \%$ & $8 \%$ \\
\hline
\end{tabular}

\subsection{Adaptivity}

Adaptivity, in essence, means the ability to change the labelling as a response to some independent variable, called an adaptation driver. Adaptivity is a cross-cutting aspect which relates to the content of the message, its presentation, and the switch between the different styles of controlling the labelling process. We here discuss the adaptation drivers and what they affect in the labelling elements.

- Stage of addiction. The grade of DA should be taken into account when deciding the friendliness of the language used in the message, gain vs. loss framing, the medium of presentation, the persistent of the message, and ultimately the amount of control given to the software. It is not always straightforward to measure the stage of addiction. Time spent and hasty actions are not the only measure to consider. Participants mentioned cases where people could leave the windows open without being really engaged with it. Other usages could relate in part to their daily work updates. That is, the weight of the usage time should be different.

- Computing device. People demonstrate different addiction patterns depending on the computing device they use. For example while checking a mobile phone frequently is just a way to let time pass in the waiting room, refreshing a website on a $\mathrm{PC}$ in the same way would not be often a typical use. The computing device also affects the feasibility of each way to deliver the warning message. For example, much illustration is unlikely recommended on mobile devices. The context of use in the case of mobile devices is a factor while it is moderately an important factor in the case of stationary access. For example, a warning would need to check 
whether a person is walking when mobile phones are used to estimate the risk and issue a suitable label. The same check would not be needed in the case of a PC.

- Time. The time of usage is also an important factor to consider when adjusting the label. Using social networks at night is typically more acceptable than using during the day. Weekends and holidays are also known for leisure activities including the infotainment and relatively higher usage of social media.

- Social context. It seems that one of the important factors is also the seasonal engagement with software which may not be a sign of excessive usage. E.g. in a festival time people tend to use social media more and sometime in exaggerated style. This is analogous to some tolerance in food and drinking style in a festival period.

- Personal profile. This includes mainly the age. In the case of children, the label would may even need to be addressed, or at least a copy of it, to some guardian, though again this introduces further ethical and perhaps legal dimensions. It appears there may also be some personality traits that lead to the acceptance of the language used in the label and the persistent nature of that label. Although we cannot confirm any correlation without further investigation, we could make few observations. For example, some participants liked the idea that the label is persistent so that they take it seriously while others did not like that. Some participants preferred to be called by their own names as a sign of respect while others saw that irrelevant especially when it is coming from software. The presentation as a pop-up seems to be necessary for certain people while others prefers a less direct style such as the light and the colour based alert. Although these settings are adjustable by the user, an intelligent inference and a mapping of individual users to Personas seems to be necessary at least to suggest a default option to users.

Table 2 shows the survey results on the same aspect.

Table 2. Users view on the adaptivity dimensions DA label

\begin{tabular}{|l|c|c|c|c|c|}
\hline \multicolumn{1}{|c|}{ Statement } & SA & A & N & D & SD \\
\hline $\begin{array}{l}\text { The progress or stage of my addictive or excessive or hasty use } \\
\text { (e.g., by changing the language and frequency accordingly) }\end{array}$ & $25 \%$ & $67 \%$ & $7 \%$ & $0 \%$ & $1 \%$ \\
\hline $\begin{array}{l}\text { The type of devices I am using (e.g., my usage patterns and } \\
\text { preferences on labelling may differ between mobile devices and } \\
\text { computers) }\end{array}$ & $24 \%$ & $61 \%$ & $8 \%$ & $7 \%$ & $0 \%$ \\
\hline $\begin{array}{l}\text { The time aspect (e.g., weekends and night time are probably } \\
\text { peak time for using social software but it does not mean my } \\
\text { usage would be excessive) }\end{array}$ & $29 \%$ & $49 \%$ & $13 \%$ & $8 \%$ & $1 \%$ \\
\hline $\begin{array}{l}\text { My social context (e.g., in holidays or parties, one may post } \\
\text { more on a social network) }\end{array}$ & $15 \%$ & $53 \%$ & $18 \%$ & $11 \%$ & $3 \%$ \\
\hline $\begin{array}{l}\text { My personal profile (e.g., age, profession and sociability are all } \\
\text { factors when judging whether it is an addictive use) }\end{array}$ & $18 \%$ & $40 \%$ & $22 \%$ & $17 \%$ & $3 \%$ \\
\hline
\end{tabular}




\section{$4 \quad$ Engineering Challenges for DA Labelling Requirement}

Labelling is fundamentally a requirements engineering problem. It is an ethical and professional practice requirement and, given the clear consequences in certain cases and feedback from users groups it may be eventually enshrined in law [12]. In terms of beneficiaries, and in addition to the users themselves, software developers also gain potential benefits from implementing labelling, regardless of the existence of laws and social norms. As we explained in Section 3.4, users tend to trust the software more when it cares about their healthy usage and trust would typically increase their loyalty level. Obviously, we still need to study the fine line between carefulness and annoyance and how software should reason about that. Health and Welfare Services, both public and private sector, are further examples of "off-stage" actors where there is already growing interest in the possibility of using addiction-aware software to maintain the mental and social-wellbeing of users [13].

Our study identified four paradoxical requirements of users which DA labelling process would need to handle:

- Control vs. autonomy paradox: users would like software to be autonomous in measuring their usage and issuing the labels. At the same time, they would like to have a control over the process. Almost all the survey participants who chose to give the autonomy to software chose also to control certain aspects over the labelling (Section 3.3). While the comfort is the motivation for the first, the effort needed for the second is clearly an obstacle to the design of such software. We proposed the notion of calibrated agreement as a sort of natural selection to elect the best control strategies between users and software over time. While this could also be the case for other kinds of requirements, e.g. privacy and security, there is an extra motivation in the case of DA labelling. This is mainly due to the supervision and mentorship nature of this requirement unlike the classical case where users use a sort of control panel to specify their software settings. Also, DA labels are not necessarily generated and sent by software but could also be sent by social entities, individuals and groups, which would add a social dimension to the control.

- Appreciation vs. Annoyance paradox: users stated that their level of trust in the software would increase when it offered the labelling service. This will be still true even if they do not like this service all the time. From the interview comments and also those provided in the text entries in the survey, we observed that users would still have a complex set of requirements on how labelling should work, which makes it relatively easy for the labelling to become an annoyance. While this relates to the previous paradox in part, software industries would be still sceptical about introducing a label and unsure of at what stage this should be done. Management of the change when introducing the labels to existing software is one further challenge. Introducing it with new software, as one of the interviewees said, may be a better option as "if it appears as part of the terms and conditions, one would both appreciate and be prepared to see it in the future".

- Being cared vs. privacy. Seven of the interviewees and $36 \%$ of the survey participants found the idea of socially generated labels interesting and would want to make the control of software usage a sort of 'enjoyable' social activity. Knowing 
that more information about their usage will help others to give more meaningful DA labels, though privacy concerns are still clearly an obstacle. Interestingly users tended to have different preferences depending on whether the carer was 'just' software or a social entity. The space for negotiation and changing perception in sharing as a mutual care and community-related activity is still worthy of further work. This is particularly true given the novelty of the concept.

- Individual vs. collective paradox. Users indicated that the reason why they may excessively be online is mainly because others are online and that they feel they might be missing something if they stop. We may think of the DA label in this case as a label directed to a community rather than to an individual all the times. However, the border between the two is blurred.

DA labelling requires a multidisciplinary research by nature. A requirements engineer would need to understand how users and other stakeholders like these labels to be designed, deduced and presented for specific software. While these decisions may still relate to the nature of that software and its design objectives, they would not be achievable simply by consulting users and probably the entity who will own the software. These decisions require foundational research which is in part a requirements engineering research. Amongst other areas, this should include the following:

- Generating factually correct DA labels content, e.g. proven consequences. While this is clear in substance addiction, the research on DA is still in the early stages.

- Laws and code of ethics. It is becoming regular news on the media that the use of certain software features, e.g. the Selfie and games, is causing harm to people. The laws and also software engineering code of ethics would need to be updated to handle the peculiarities of the addictive nature of software. DA labels are still seen an option rather than a norm or even professional practice.

- Fuzziness and metrics. Monitoring usage and judging its addictive nature will need metrics that manage to represent a complex set of behaviours, particularly given the fuzzy nature of the concept. For example, $78 \%$ of users wanted software to appreciate that in holidays and at certain time of the day their usage could follow a different pattern to their normal levels. Similarly, as with physical addictions, such as to alcohol, some people have different thresholds of what an excessive use is, and this is further complicated by the fact that the amount, say consumed, is not always the sole indicator of dependence. For this reason, only $11 \%$ of our users disagreed that solely measuring their usage is never going to be sufficient as a predictor of addiction, and, therefore, DA labels would need to be less confirmative; one alternative being some kind of individual calibration.

- Alignment. We can view the DA labelling as an alignment problem in which the software has a requirement to align with health and social care regulations. However, although we use the term "addiction", the phenomenon of the excessive, impulsive and compulsive usage of software is still not formally considered a sort of addiction or disorder. We would still need a multidisciplinary research to come up with metrics for the healthy usage and what properties to ensure in the software design to maintain it. 
In the case of DA labelling, and DA in general, requirements elicitation has a particularly private nature. To add weight to our argument, our pre-selection survey enabled us to interview only those who declared a sort of addictive usage. However, during the interview, participants moderated quite significantly their description of their usage style. People would typically feel uncomfortable declaring their true usage style when they are identified. This will call to a requirements elicitation which is a mixture of psychology and software engineering practice, in order to tackle DA labelling.

\section{Conclusions}

We advocated the need for Digital Addiction labels and studied how this should be implemented from a user's perspective through an empirical study. The vast majority of participants, $+80 \%$, confirmed it is potentially a good idea to aid them regulate their usage style. Besides the results which one would intuitively speculate about the content and presentation (time spent and clock, etc.), our study led to interesting findings. These are mainly in the area of the paradoxical need for freedom and being supervised in the same time, the novel ways of generating and communicating the label, the private nature of this requirement, the need for metrics for addictive usage, to name a few. Our future will delve into the details of each of these challenges from the perspectives of both requirements engineering and cyber-psychology.

\section{Acknowledgment}

The research was supported by an FP7 Marie Curie CIG grant (the SOCIAD Project) and by Bournemouth University through the Fusion Investment Fund.

\section{References}

1. Nyland, R., Marvez, R., Beck, J.: MySpace: Social networking or social isolation? In: AEJMC Midwinter Conference (pp. 23-24), 2007.

2. Kirschner, P.A., Karpinski, A.C.: Facebook and academic performance. Comput. Hum. Behav. 2010, 26, 1237-1245.

3. Barker, V.: Older adolescents' motivations for social network site use: The influence of gender, group identity, and collective self-esteem. CyberPsychol. Behav. 2009, 12, 209213.

4. Ellison, N.B., Steinfield, C., Lampe, C.: The benefits of Facebook "friends": Social capital and college students' use of online social network sites. Comput-Mediat. Comm. 2007, 12.

5. Kuss, D. J., Griffiths, M. D.: Online social networking and addiction - a review of the psychological literature. Environmental research and public health 8.9 (2011): 3528-3552.

6. Young, K.: Internet addiction: Evaluation and treatment. Student Brit. Med.1999, 7, 351352

7. Griffiths, M. D.: Components model of addiction within a bio-psychosocial framework. J. Subst. Use 2005, 10, 191-197.

8. Alrobai, A., Phalp, K., Ali, R.: Digital Addiction: a Requirements Engineering Perspective. In: The 20th International Working Conference on Requirements Engineering: Foundation for Software Quality (REFSQ 2014). Essen, Germany. April 2014. 
9. Jiang, N., Ali, R.: On the Delivery of Recommendations in Social Software: a User`s Perspective. In: The 5th International Conference on Human-Centred Software Engineering (HCSE 2014). Paderborn, Germany. September 16th-18th, 2014.

10. Zichermann, G., Cunningham, C.: Gamification by design: Implementing game mechanics in web and mobile apps. " O'Reilly Media, Inc., 2011.

11. Tversky, A., Kahneman, D.: Advances in prospect theory: Cumulative representation of uncertainty. Journal of Risk and Uncertainty, 5, 297-323. 1992.

12. Da Zhan, J., Chan, H.C.: Government Regulation of Online Game Addiction. Communications of the Association for Information Systems 30 (2012).

13. Kim, K.: Internet addiction in Korean adolescents and its relation to depression and suicidal ideation: a questionnaire survey. Intl. journal of nursing studies 43.2 (2006): 185-192.

14. Thaler, R.H, Sunstein, C.R.: Nudge: Improving Decisions About Health, Wealth and Happiness. Yale University Press (2009).

15. Ewing, J. A.: Detecting alcoholism: the CAGE questionnaire. Jama 252.14 (1984): 19051907. 